\title{
THE GENETICAL BASIS OF HETEROKARYON INCOMPATIBILITY IN ASPERGILLUS NIDULANS
}

\author{
J. L. JINKS and M. GRINDLE \\ A.R.C. Unit of Biometrical Genetics, Department of Genetics, \\ University of Birmingham
}

\section{INTRODUCTION}

Received 2r.iii.63

IN general, isolates of Aspergillus nidulans from widely scattered sources will not form heterokaryons with one another when grown together (Grindle, 1963a). Isolates from more restricted areas, however, are able to form heterokaryons occasionally. On their ability or inability to form heterokaryons such isolates can be classifised into a number of compatibility groups (Grindle, $1963 b$ ). Different isolates belonging to the same group form heterokaryons with one another as readily as do pairs of strains derived from the same isolate by mutation. Isolates belonging to different groups, on the other hand, do not in general form heterokaryons when grown together. There are, however, exceptions. Some combinations of isolates from different compatibility groups will form heterokaryons but the proportion of cells produced which contain both kinds of nuclei is extremely low, being a small percentage of that produced by fully compatible combinations.

We cannot analyse the basis of the complete incompatibility between isolates because heterokaryon formation is an essential preliminary to a genetic analysis in this fungus (Pontecorvo, 1953). It is possible, however, to investigate the genetical control of the reduced compatibility shown by some combinations of isolates from different compatibility groups. But in these instances the frequency of heterokaryotic cells is so low (approximately $0 \cdot 1$ per cent.) that an analysis of the progeny from crossed perithecia is out of the question. Alternative methods have, therefore, been used which are indirect but both practicable and unambiguous in their results.

\section{MATERIAL AND METHOD}

The material is strains of isolates 2 and 26 . These were obtained from the same locality but belong to two different compatibility groups; isolate 2 belongs to group A and isolate 26 to group B (Grindle, $1963 b$ ). Eight pairs of isolates in groups $A$ and $B$, including 2 and 26 , produce heterokaryotic cells when they meet, with a very low frequency.

The frequency of heterokaryotic cells produced by a pair of strains is estimated from the frequency of conidiophores which contain nuclei of both strains and hence produce chains of asexual spores of two kinds. This frequency is regularly 20 to 40 times greater for pairs of strains belonging to the same compatibility group than for pairs of partially compatible strains which belong to different groups.

The medium, method of culture and the scoring of the frequency of heterokaryotic conidiophores are as described by Grindle (1963a and $1966_{3} b$ ). 


\section{HETEROKARYON TEST}

Differences between the component strains of a heterokaryon which are dependent on chromosomal gene differences usually emerge unaltered from the heterokaryotic association. Differences which are dependent on extrachromosomal factors, on the other hand, are either lost or show reassortment between the components as a result of a heterokaryotic association (Jinks, I954, I958, I 96 I). To distinguish between a chromosomal and extrachromosomal determination of low compatibility the following combination of strains were grown in mixed culture: $2 \mathrm{wY}$ and $26 \mathrm{WY}, 2 \mathrm{WY}$ and $26 \mathrm{Wy}$, $2 \mathrm{wY}$ and $26 \mathrm{Wy}, 2 \mathrm{wY}$ and $2 \mathrm{WY}$ and $26 \mathrm{Wy}$ and $26 \mathrm{WY}(\mathrm{W}: \mathrm{w}$ being green versus white asexual spores and $\mathrm{Y}: \mathrm{y}$ green versus yellow asexual spores). Along the lines of contact between the pairs of strains heterokaryotic conidiophores are produced which are recognised by the white and green, yellow and green or white and yellow chains of asexual spores they bear. The three combinations of strains involving one from isolate 2 and one from isolate 26 produced about 0.2 per cent. of such conidiophores while those involving two strains of isolate 2 or two of isolate 26 produced about 4 to 6 per cent.

The two strains were recovered from the heterokaryotic association by germinating the spores produced by the heterokaryotic conidiophores. For example, the heterokaryotic conidiophores produced by strains $2 \mathrm{WY}$ and $26 \mathrm{WY}$ gave both white and green chains of spores which germinated to give white ( $2 \mathrm{wY}$ ex-heterokaryon) and green (26WY ex-heterokaryon) sporing colonies respectively. Individual white sporing individuals were then classified for their heterokaryon compatibility with strains $2 \mathrm{WY}$ and $26 \mathrm{WY}$. Similarly, individual green sporing colonies were classified for their heterokaryon compatibility with strains $2 \mathrm{wY}$ and $26 \mathrm{Wy}$. From all three combinations of strains of isolates 2 and 26 a total of ${ }_{1} 36$ colonies of strains $2 \mathrm{wY}$ and $2 \mathrm{WY}$ and $\mathrm{I}_{3} 6$ colonies of strains $26 \mathrm{Wy}$ and $26 \mathrm{WY}$ were recovered from heterokaryotic association and their compatibilities determined. As controls 64 colonies of strains $2 \mathrm{WY}$ and $2 \mathrm{WY}$ and 64 colonies of $26 \mathrm{Wy}$ and $26 \mathrm{WY}$ were also recovered from the intra-isolate heterokaryons and tested similarly.

All the colonies of strains $2 \mathrm{wY}$ and $2 \mathrm{WY}$, whether from heterokaryotic association with strains of isolate 2 or of isolate 26 , proved to have the typical compatibility reaction of isolate 2 ; that is, they readily formed heterokaryotic conidiophores with strains of isolate 2 but only rarely with strains of isolate 26 . Similarly, all the colonies of strains $26 \mathrm{Wy}$ and $26 \mathrm{WY}$ whether from heterokaryotic association with isolate 26 or isolate 2 had the compatibility reaction of isolate 26 . Our conclusion, therefore, is that the compatibility difference between isolates 2 and 26 is not altered by heterokaryotic association. Thus it is presumably controlled by chromosomal genes. 


\section{THE RATE OF EXTRACHROMOSOMAL EXCHANGE}

The absence of any detectable modification in the compatibility reaction of either 2 or 26 following their heterokaryotic association suggests the absence of an extrachromosomal component of this reaction. But how sensitive is the heterokaryon test? The frequency of heterokaryotic exchange of known extrachromosomal differences varies with the nature of the difference. For example, heterokaryons between sexual and non-sexual strains of $A$. nidulans may show complete conversion of the non-sexual component to a sexual phenotype (Jinks, 1954). On the other hand, in the unstable heterokaryons between red and non-red strains of isolate I of this species, from 6 to 20 per cent. of the non-red strain acquires a red phenotype (Arlett, Grindle and Jinks, 1962). The frequency of heterokaryotic exchange also varies with the genotype of the strain used. For example, if the genotype of the non-red component favours the mutant determinant responsible for the red phenotype it shows a higher frequency of conversion to a red phenotype after heterokaryotic association with a red strain than a genotype which does not (Arlett et al., 1962; Grindle, unpublished). No estimate of the frequency of extrachromosomal exchange in strains of isolate 2 is available. However, non-red strains of isolate 26 show 9 per cent. conversion to a red phenotype following heterokaryotic association with red strains of isolate $\mathrm{I}$. This is an average result for the exchange of the extrachromosomal determinant of the red phenotype.

If we accept this estimate of 9 per cent. as the likely frequency of exchange of extrachromosomal components of isolates 2 and 26 we would have expected to recover at least 24 colonies of these isolates, from the heterokaryons between them, with the extrachromosomal components of their partner. Hence, if incompatibility is due to a difference in an extrachromosomal component we would have expected 24 colonies of isolates 2 and 26 to have had the compatibility reaction of the other isolate. This expectation, however, is based on the frequency of exchange of single factor differences between two extrachromosomal complements. If compatibility were under multifactorial control, each factor of which showed about 9 per cent. reassortment in a heterokaryon, the expected frequency of colonies of isolates 2 and 26 with the compatibility reaction of the other isolate would be lower. In fact it might be too low to be detected by a heterokaryon test. A selection experiment was, therefore, undertaken in order to test the possibility of a multifactorial extrachromosomal control of compatibility reaction.

\section{SELECTION FOR INCREASED COMPATIBILITY}

We wish to test the possibility that the compatibility differences between isolates 2 and 26 are due to the cumulative effects of a number 
of extrachromosomal factors each of small effect (since they individually remained undetected in the heterokaryon test) and each showing about Io per cent. exchange between the components of a heterokaryon. To carry out the test (I) we require an opportunity for the exchange of extrachromosomal factors between isolates 2 and 26 , (2) we need to detect slight changes in compatibility resulting from such exchanges, and (3) we need to accumulate such changes by successive cycles of selection for colonies showing the highest level of inter-isolate heterokaryon compatibility. To meet these requirements the following design was adopted. Strains of isolates 2 and 26 differing in the colour of their spores were grown in mixed culture and the spores produced by the heterokaryotic conidiophores were recovered. The spores were germinated and the colonies produced were classified into homokaryons 2 and 26 on the basis of their spore colour. A random sample of 20 colonies of homokaryon 2 and 20 of homokaryon 26 were scored for their heterokaryon compatibility with suitably marked strains of the other isolate by growing them in mixed culture and counting the frequency of heterokaryotic conidiophores. On the basis of this frequency, in four replicate tests, the most compatible of the 20 colonies of homokaryons 2 and 26 were selected. From the most compatible combination further samples of spores from heterokaryotic conidiophores were recovered, germinated and the resulting colonies classified into homokaryons 2 and 26 . The whole cycle of testing the compatibility, selection and extraction from the most compatible combination was repeated four times. The colonies of homokaryons 2 and 26 obtained from this final extraction were scored for their compatibility reaction against strains of both isolates 2 and 26 . The homokaryon 2 colonies all showed the compatibility reaction of the original isolate 2. Similarly, homokaryon 26 colonies all showed the compatibility reaction of the original isolate 26 . Thus the repeated opportunities for the strains of these isolates to exchange extrachromosomal factors and repeated selection for those colonies of the strains which appeared to have above average heterokaryon compatibility, produced no response.

\section{CONCLUSIONS AND SUMMARY}

The failure of compatibility reaction to change or to show reassortment when two strains with different reactions are associated in a heterokaryon suggests that compatibility reaction is controlled by chromosomal genes. This conclusion is confirmed by the failure to break down a compatibility difference between two isolates by repeated heterokaryotic association accompanied by selection for increased compatibility. The situation in $A$. nidulans is therefore similar to that reported in Neurospora crassa. In this latter species heterokaryon compatibility is controlled by a number of chromosomal loci. In Neurospora, however, heterokaryosis is not a step in outcrossing and 
hence strains which are heterokaryon incompatible may still be sexually compatible. The consequences of the system of heterokaryon incompatibility are, therefore, potentially quite different in Aspergillus and Neurospora.

\section{REFERENCES}

ARLETT, C. F., JINKS, J. L., AND GRINDLE, M. I962. The "red " cytoplasmic variant of Aspergillus nidulans. Heredity, 17, 197-209.

GRINDLE, M. I963. Heterokaryon compatibility of unrelated strains in the Aspergillus nidulans group. Heredity, I8, I9I-204.

GRINDLE, M. I963. Heterokaryon compatibility of closely related wild isolates of Aspergillus nidulans. Heredity, I8, 397-405.

JINKs, J. L. 1954. Somatic selection in fungi. Nature, 174, 409.

Jinks, J. L. 1958. Cytoplasmic differentiation in fungi. Proc. Roy. Soc. B, I48, 314-32I.

JINks, J. L. I963. Cytoplasmic inheritance in fungi, pp. 325-354. Methodology in Basic Genetics. Holden-Day Inc. San Francisco.

pontecorvo, G. 1953. The genetics of Aspergillus nidulans. Advance. Genet., 5, I 4 I -238 . 